\title{
FREE BOUNDARY PROBLEMS FOR PARABOLIC EQUATIONS
}

\author{
BY AVNER FRIEDMAN
}

1. One dimensional problems. Denote by $D_{i}(T)(1 \leqq i \leqq k)$ a 2-dimensional domain bounded by two curves $x=s_{i-1}(t), x=s_{i}(t)$ where $0<t<T$, and by the line segments $t=0, b_{i-1}<x<b_{i}$ and $t=T$, $s_{i-1}(T)<x<s_{i}(T)$. Here $s_{i-1}(t)<s_{i}(t), s_{0}(t) \equiv b_{0}, s_{k}(t) \equiv b_{k}$ where $b_{0}, b_{k}$ are constants. Let

$$
L_{m} u \equiv a^{m}(x, t) \frac{\partial^{2} u}{\partial x^{2}}+b^{m}(x, t) \frac{\partial u}{\partial x}+c^{m}(x, t) u-\frac{\partial u}{\partial t} \quad(m=1,2)
$$

be parabolic operators with smooth coefficients and with $c^{m}(x, t) \leqq 0$. Suppose, for definiteness, that $k$ is an even number. Consider the following problem: Find such curves $s_{1}, \cdots, s_{k-1}$ and functions $u_{1}, u_{2}$, that

(1.5) either $u_{1}=g_{1} \quad$ or $\quad \lambda_{1} \frac{\partial u_{1}}{\partial x}+\mu_{1} u_{1}=g_{1} \quad$ for $x=b_{0}, \quad 0<t<T$, (1.6) either $u_{2}=g_{2}$ or $\quad \lambda_{2} \frac{\partial u_{2}}{\partial x}+\mu_{2} u_{2}=g_{2} \quad$ for $x=b_{k}, \quad 0<t<T$,

$$
\begin{gathered}
u_{1}=u_{2}=\Phi\left(\frac{\partial u_{1}}{\partial x}, \frac{\partial u_{2}}{\partial x}, s_{i}, \frac{d s_{i}}{d t}\right) \quad \text { on } x=s_{i}(t), \\
0<t<T \quad(1 \leqq i \leqq k-1), \\
\Psi\left(u_{1}, u_{2}, \frac{\partial u_{1}}{\partial x}, \frac{\partial u_{2}}{\partial x}, s_{i}, \frac{d s_{i}}{d t}\right)=0 \quad \text { on } x=s_{i}(t), \\
0<t<T \quad(1 \leqq i \leqq k-1) ;
\end{gathered}
$$

An address delivered at the Ann Arbor meeting of the American Mathematical Society on November 29, 1969, by invitation of the Committee to Select Hour Speakers for Western Sectional Meetings; received by the editors April 9, 1969.

AMS 1969 subject classifications. Primary 3562, 3578.

Key words and phrases. Parabolic equations, free boundary, melting of solids, stability of solutions, asymptotic behaviour, weak solution. 
here $f_{i}, h_{i}, \lambda_{i}, \mu_{i}, g_{i}, \Phi$ and $\Psi$ are given functions. Such a problem is called a $k$-phasefree boundary problem. The curves $x=s_{i}(t)(1 \leqq i \leqq k-1)$ are called free boundaries.

One can also formulate the analogous problem in case $k$ is odd, in case $s_{0}(t)$ and $s_{k}(t)$ are given nonconstant functions, and also in case $b_{0}=-\infty$ or $b_{k}=\infty$. The problem can further be generalized by considering $k$ parabolic operators $L_{m}$ and replacing (1.1), (1.2) by

$$
L_{m} u_{m}=f_{m} \quad \text { in } D_{m}(T) \quad(1 \leqq m \leqq k) .
$$

One then makes the obvious changes in the other conditions (1.3)(1.8). Finally, in some problems, $\Phi$ or $\Psi$ are not pointwise functions of their variables but are rather functionals.

Some natural questions arise regarding the above formulated free boundary problem:

(i) Does there exist a unique solution for any $T$ ?

(ii) Does the solution depend monotonically upon the initial and boundary conditions?

(iii) Is the solution a continuous function of the data, in certain norms?

(iv) Does the solution have an asymptotic behaviour as $t \rightarrow \infty$ ?

(v) Often the parabolic equations or the boundary conditions at the free boundary depend on a parameter $\alpha$. How does the solution depend on $\alpha$ ?

The most studied free boundary problem is the Stefan problem. Here $u_{1}$ represents the temperature of water and $u_{2}$ represents the temperature of ice. The problem is then a model of the process of melting. In the condition (1.7), $\Phi \equiv 0$. The condition (1.8) reduces to the equation of conservation of energy:

$$
\alpha_{1} \partial u_{1} / \partial x-\alpha_{2} \partial u_{2} / \partial x=d s_{i} / d t
$$

where $\alpha_{1}, \alpha_{2}$ are positive (constants or functions). We further have, $g_{1} \geqq 0, h_{1} \geqq 0, f_{1} \leqq 0, g_{2} \leqq 0, h_{2} \leqq 0, f_{2} \geqq 0$. The literature on this problem is quite extensive. We refer to the books of Friedman [1] and Rubinstein [2] and to the references given there. Some more recent work can be found in [3]-[10]; see also Kyner [11] for a nonlinear Stefan problem. Much of the work is concerned with the one phase problem, that is, $k=2$ and $u_{2} \equiv 0, b_{k}=\infty$. In this case the free boundary $x=s_{1}(t)$ is a monotone function.

In most of the papers on the Stefan problem the authors reduce the problem to that of solving nonlinear integro-differential equations of Volterra type (for either $s_{i}(t)$, or $d s_{i}(t) / d t$, or $\left.\partial u_{j}\left(s_{i}(t), t\right) / \partial x\right)$. Then they proceed to prove the existence of a solution in a small time inter- 
val. In order to derive the existence of a global solution, it is necessary to obtain an a priori bound on the first derivatives $\partial u_{i}(x, t) / \partial t$ for $t<\tau$; the bound must be a bounded function of $\tau$. Such a bound has been derived for the one phase problem [12]; for the $k$-phase problem this bound has been derived only for small data [6].

Results in the direction of problem (iii) are obtained from the proof of existence of the solution. Problem (ii) has been solved by Rubinstein [13] and Cannon and Hill [3], [4]. Problem (iv) for the one phase case was treated by Friedman [14] and Cannon and Hill [5]. For $k=2$ Rubinstein [13] established the asymptotic convergence of classical solutions; however, it is not known whether such solutions do indeed exist (except for small data).

The following problems remain unsolved by the above classical approach (of reduction to a nonlinear integro-differential equation) and by variants of it:

Problem A. Existence of a global solution for the $k$-phase Stefan problem, $k \geqq 2$.

Problem B. If the boundary data converge (in a suitable norm) as $t \rightarrow \infty$, does the solution have a limit as $t \rightarrow \infty$ ?

These problems have been solved in Friedman [15], where the concept of a solution is "nearly" classical. The methods depend however on techniques developed for the $n$-dimensional Stefan problem. We shall therefore consider first (in \$2) the latter problem, and then (in §3) return to Problems A, B.

We conclude this section with a few references to non-Stefan problems in one dimension. In [16], [17] the free boundary problems differ from the Stefan problem in the form of the function $\Psi$ (occurring in (1.8)). There is also a small parameter entering into the problem. It is shown that the (global) solution exists and that it has a power series expansion in $\alpha$. Furthermore, asymptotic formulas are derived for the free boundary $x=s(t, \alpha)$ as $t \rightarrow \infty, \alpha \rightarrow 0$. Kruzhkov [18] has considered a one phase free boundary problem arising from the impact of a viscoplastic rod on a rigid obstacle. Here the conditions at the free boundary $x=s(t)$ are:

$$
\partial u / \partial x=0, \quad u=A(s, t)
$$

where $A$ is some integral operator. He proved the uniqueness and the monotone behaviour of the solution. However, the existence of a solution is not known. Fulks and Guenther [19] have recently considered a two phase free boundary problem of two incompressible fluids in a compressible porous medium. Here the conditions at the free boundary are: 


$$
u_{1}=u_{2}=d s / d t, \quad H+\gamma \partial u_{1} / \partial x=\lambda \partial u_{2} / \partial x
$$

where $H$ is some functional of $u_{1}$. They proved the existence and uniqueness of a global solution.

2. $n$-dimensional Stefan problem. We begin with a classical formulation of the problem. Let $G$ be a bounded domain in $R^{n}$, whose boundary consists of connected manifolds $M_{i}, i=1,2, \cdots, k_{1}, k_{1} \geqq 2$. Let

$$
\partial_{1} G=\bigcup_{i=1}^{k_{0}} M_{i}, \quad \partial_{2} G=\bigcup_{i=k_{0}+1}^{k_{1}} M_{i} .
$$

We assume that $\partial_{1} G$ and $\partial_{2} G$ are both nonempty and (for definiteness) that $\partial_{2} G$ contains the outer boundary of $\partial G$. Write

$G(t)=\{(x, t) ; x \in G\}, \quad \Omega_{T}=\bigcup_{0<t<T} G(t), \quad \partial_{i} G(t)=\left\{(x, t) ; x \in \partial_{i} G\right\}$.

The free boundary will consist of a set $\Gamma=\{\Gamma(t), 0<t<T\}$ where $\Gamma(t)$ is a manifold lying in $G(t)$. Denote by $G_{i}(t)$ the open set bounded by $\partial_{i} G(t)$ and $\Gamma(t)$ and let

$$
\Omega_{T, i}=\bigcup_{0<t<T} G_{i}(t)
$$

Consider the parabolic equations

$$
\begin{array}{ll}
\alpha_{1} \frac{\partial u_{1}}{\partial t}=\Delta u_{1}+c_{1} u_{1} \quad \text { in } \Omega_{T, 1} & \left(c_{1} \leqq 0\right), \\
\alpha_{2} \frac{\partial u_{2}}{\partial t}=\Delta u_{2}+c_{2} u_{2} \quad \text { in } \Omega_{T, 2} & \left(c_{2} \leqq 0\right)
\end{array}
$$

where $\alpha_{1}, \alpha_{2}$ are positive constants, the initial conditions

$$
\begin{array}{lll}
u_{1}(x, 0)=h_{1}(x) & \text { in } G_{1}(0) & \left(h_{1} \geqq 0\right), \\
u_{2}(x, 0)=h_{2}(x) & \text { in } G_{2}(0) & \left(h_{2} \leqq 0\right),
\end{array}
$$

and the boundary conditions

$$
\begin{aligned}
& u_{1}=g_{1} \quad \text { on } \partial_{1} G \times(0, T) \quad\left(g_{1} \geqq 0\right), \\
& u_{2}=g_{2} \text { on } \partial_{2} G \times(0, T) \quad\left(g_{2} \leqq 0\right) \text {. }
\end{aligned}
$$

Suppose $\Gamma(t)$ is given by $\Phi(x, t)=0$. Then we impose the following conditions:

$$
\begin{gathered}
u_{1}=u_{2}=0 \text { on } \Gamma, \\
\alpha_{1} \nabla_{x} u_{1} \cdot \nabla_{x} \Phi-\alpha_{2} \nabla_{x} u_{2} \cdot \nabla_{x} \Phi=\alpha \Phi_{t} \text { on } \Gamma
\end{gathered}
$$


where $\alpha$ is a positive constant.

In general, when $n \geqq 2$, a classical solution $\left(u_{1}, u_{2}, \Phi\right)$ of the Stefan problem (2.1)-(2.8) does not exist. Indeed, a large portion of the free boundary may disappear all of a sudden when a thin island of ice is surrounded by warm water.

We proceed to define the concept of a weak solution. Set

$$
\begin{aligned}
g=g_{i} / \alpha_{i} & \\
h=h_{i} / \alpha_{i} & \text { on } \partial_{i} G \times(0, T), \\
a(u)=\alpha_{1} u & \text { if } u>0, \\
=\alpha_{2} u-\alpha & \text { if } u<0 .
\end{aligned}
$$

Let $u(x, t)$ be a measurable function in $\Omega_{T}$. A bounded measurable function $\gamma(x, t)$ is denoted by $a(u(x, t))$ if

(i) at each point $(x, t)$ where $u(x, t) \neq 0, \gamma(x, t)=a(u(x, t))$, and

(ii) at each point $(x, t)$ where $u(x, t)=0,-\alpha \leqq \gamma(x, t) \leqq 0$.

Definition. A weak solution of (2.1)-(2.8) is a bounded measurable function $u(x, t)$ in $\Omega_{T}$ for which there is a function $a(u(x, t))$ such that the following is true:

$$
\begin{aligned}
\iint_{\Omega}\left[u(\Delta \phi+c \phi)+a(u) \phi_{t}\right] & d x d t \\
= & \int_{0}^{T} \int_{\partial G} g \frac{\partial \phi}{\partial \nu} d S_{x} d t-\int_{G(0)} a(h) \phi d x
\end{aligned}
$$

for any $\phi$ such that $\nabla_{x} \phi, \nabla_{x}^{2} \phi, \phi_{t}$ are continuous in $\bar{\Omega}_{T}$ and $\phi=0$ on $G(T)$ and on $\partial G \times(0, T)$. Here $c=c_{1} / \alpha_{1}$ at the points where $u>0$ and $c=c_{2} / \alpha_{2}$ at the points where $u<0$.

The above definition is due to Kamenomostskaja [20]. One can easily show that a classical solution is a weak solution. Conversely, a weak solution for which the null set is a smooth manifold and which is sufficiently smooth on each side of this manifold is a classical solution.

Using a finite difference scheme, Kamenomostskaja [20] proved the existence of a weak solution and the bound

$$
\iint_{\Omega}\left|\nabla_{x} u\right|^{2} d x d t \leqq C .
$$

In [21] Friedman used a simpler method based on energy inequalities and a "regularization" of the problem, to prove the following stronger result:

$\left(R_{1}\right)$ Under some standard regularity assumptions on the data, there exists a weak solution $u$ satisfying 


$$
\int_{G}\left|\nabla_{x} u(x, t)\right|^{2} d x \leqq C \quad(0 \leqq t \leqq T) .
$$

Kamenomostskaja [20] showed that the weak solution is unique. This is contained in the following stability theorem proved by Friedman [21]:

$\left(\mathrm{R}_{2}\right)$ Let $u$ and $u$ be two weak solutions corresponding to data $h, g$ and $h, g$ respectively. Let $\Psi$ and $\Psi$ be any extensions of $g$ and $\hat{g}$ into $\Omega_{T}$. Then

$$
\begin{aligned}
& \int_{0}^{T} \int_{G}(a-u)[a(\hat{u})-a(u)] d x d t \\
& \leqq B \int_{G(0)}(\hat{h}-h)^{2} d x+\int_{0}^{T} \int_{G}\left[(\hat{\Psi}-\Psi)^{2}+\left|\nabla_{x}(\hat{\Psi}-\Psi)\right|^{2}\right] d x d t,
\end{aligned}
$$

where $B$ is a constant independent of $T$ (provided $c(x, t)$ is "well behaved" as $t \rightarrow \infty)$.

Combining (2.10), (2.11) and using Sobolev type inequalities, the following result is obtained:

$\left(\mathrm{R}_{3}\right)$ Assume that $g(x, t)$ is convergent to $g_{\infty}(x)$ as $t \rightarrow \infty$, in the following sense:

$$
\sup \left[|g|+\left|D_{x} g\right|+\left|D_{t} g\right|+H_{\eta}\left(D_{x} g\right)\right]<\infty
$$

where $D_{x} g$ is the gradient of $g$ restricted to $\partial G \times\{t\}$, and $H_{\eta}$ is a Hölder coefficient for some exponent $\eta>0$, and

$$
\int_{1}^{\infty} \int_{\partial G}\left[\left(g_{t}\right)^{2}+\left(g-g_{\infty}\right)^{2}+\left|D_{x}\left(g-g_{\infty}\right)\right|^{2}\right] d S_{x} d t<\infty .
$$

Then, for $n \geqq 2$ and $p<2 n /(n-2)$,

$$
\int_{G}|u(x, t)-w(x)|^{p} d x \rightarrow 0 \text { if } t \rightarrow \infty,
$$

and, for $n=1$,

$$
u(x, t) \rightarrow w(x) \text { as } t \rightarrow \infty, \quad \text { uniformly in } x \in G .
$$

Here $w(x)$ is the harmonic function in $G$ satisfying $w=g_{\infty}$ on $\partial G$.

The method of construction of the solution in [21] yields the following result:

$\left(\mathrm{R}_{4}\right)$ If $h \leqq \hat{h}, g \leqq \hat{g}$ then $u \leqq \hat{a}$.

Definition. Let $u$ be a weak solution. The set $S$ where $-\alpha<a(u(x, t))<0$ is called the generalized free boundary. It is determined up to a set of measure zero. 
If $h_{2} \equiv 0, g_{2} \equiv 0$ and if $\partial G_{2}$ is empty (that is, the ice has temperature $u_{2} \equiv 0$ and it extends to $\infty$ ) then we call the Stefan problem a one phase problem. For such a problem it was proved in [21] that

$$
\text { int } S=\varnothing \text {. }
$$

It is still an open question whether or not

$$
\text { meas. } S=0 \text {. }
$$

As for the general $n$-dimensional Stefan problem, it is not known whether (2.14) is true.

In [21] the following asymptotic bounds are derived on the location of the generalized free boundary for the one phase problem:

$\left(R_{8}\right)$ Assume that

$$
\begin{array}{ll}
\gamma<\frac{g_{1}(x, t)}{1+t^{(n-2) / 2}}<\gamma^{\prime} & \text { if } n \geqq 3, \\
\gamma<\frac{g_{1}(x, t)}{\log (2+t)}<\gamma^{\prime} & \text { if } n=2, \\
\gamma<g_{1}(x, t) \quad<\gamma^{\prime} & \text { if } n=1
\end{array}
$$

where $\gamma, \gamma^{\prime}$ are positive constants. Then there exist positive constants $\beta, \beta^{\prime}$ such that if $(x, t) \in S$ then $\beta<|x| / t^{1 / 2}<\beta^{\prime}$.

3. One dimensional Stefan problem with several phases. The results of $\$ 2$ can be sharpened in the case $n=1$. In particular, one can establish (see [21]) the continuity of the weak solution $u(x, t)$. A more detailed analysis in [15] shows that the generalized free boundary can be normalized so that in the $k$-phase problem the following is true:

$\left(R_{6}\right)$ The generalized free boundary consists of a finite number of piecewise continuous curves $x=s_{i}(t)$. The points of (possible) discontinuity $\tau_{1}, \cdots, \tau_{m}$ are such that at each $\tau_{j}$ the number of phases strictly decreases. The solution $u$ is a classical solution of the parabolic equations (for water or for ice) outside the curves $x=s_{i}(t)$. On these curves, $u=0$ and the equation (1.9) is satisfied in an integrated form.

Thus we see that a global solution for the $k$-phase problem exists, and it is "nearly" classical. Problem A posed in $\$ 1$ is thus essentially solved. As for Problem B, we have already stated that (2.13) holds. In addition, using the results of $\left(\mathrm{R}_{6}\right)$ one can also show that

(3.1) $\lim _{t \rightarrow \infty} s_{i}(t)$ exists.

Thus, Problem B is completely solved. There still remains the question of deriving bounds on the rates of convergence in (2.13) and in (3.1). 
In proving $\left(R_{6}\right)$, use is made of the existence of a classical solution for small time intervals for the $k$-phase problem.

\section{REFERENCES}

1. A. Friedman, Partial differential equations of parabolic type, Prentice-Hall, Englewood Cliffs, N. J., 1964. MR 31 \#6062.

2. L. I. Rubinšteľn, Stefan problem, "Zvalgzne", Riga, 1967. (Russian) MR 36 \#5488.

3. J. R. Cannon and C. D. Hill, Existence, uniqueness, stability, and monotone dependence in a Stefan problem for the heat equation, J. Math. Mech. 17 (1967), 1-20.

4. J. R. Cannon and J. Douglas, The stability of the boundary in a Stefan problem, Ann. Scuola Norm. Sup. Pisa 21 (1967), 83-91.

5. J. R. Cannon and C. D. Hill, Remarks on a Stefan problem, J. Math. Mech. 17 (1967), 433-441. MR $36 \# 1854$.

6. J. R. Cannon, J. Douglas and C. D. Hill, A multi-boundary Stefan problem and the disappearance of phases, J. Math. Mech. 17 (1967), 21-34.

7. J. R. Cannon and C. D. Hill, On the infinite differentiability of the free boundary in a Stefan boundary, J. Math. Anal. Appl. 22 (1968), 385-397. MR 37 \#610.

8. B. Sherman, $A$ free boundary problem for the heat equation with prescribed flux at both fixed face and melting interface, Quart. Appl. Math. 25 (1967), 53-63. MR 35 \#3969.

9. - Continuous dependence and differentiability properties of the solution of a free boundary problem for the heat equation, Quart. Appl. Math. 27 (1970), 427-439.

10. J. M. Greenberg, $A$ free boundary problem for the linear heat equation, J. Differential Equations 7 (1970), 287-306.

11. W. T. Kyner, An existence and uniqueness theorem for a nonlinear Stefan problem, J. Math. Mech. 8 (1959), 483-498. MR $26 \# 1630$.

12. A. Friedman, Free boundary problems for parabolic equations. I: Melting of solids, J. Math. Mech. 8 (1959), 499-517. MR 26 \#1626.

13. L. I. Rubinšteľn, The two-phase Stefan problem on an interval with one-phase initial state of a heat-conducting medium, Latvijas valsts Univ. Zinātn. Raksti 58 (1964), no. 2, 111-148. (Russian) MR 31 \#6066.

14. A. Friedman, Remarks on Stefan-type free boundary problems for parabolic equations, J. Math. Mech. 9 (1960), 885-903. MR 26 \#1629.

15. - One dimensional Stefan problems with nonmonotone free boundary, Trans. Amer. Math. Soc. 133 (1968), 89-114. MR 37 \#3210.

16. - Free boundary problems for parabolic equations. II: Evaporation or condensation of a liquid drop, J. Math. Mech. 9 (1960), 19-66. MR 26 \#1627.

17. - Free boundary problems for parabolic equations. III: Dissolution of a gas bubble in liquid, J. Math. Mech. 9 (1960), 327-345. MR $26 \# 1628$.

18. S. N. Kružkov, On some problems with unknown boundaries for the heat conduction equation, Prikl. Mat. Meh. 31 (1967), 1009-1020=J. Appl. Math. Mech. 31 (1967), 1014-1024. MR 37 \#6618.

19. W. Fulks and R. B. Guenther, $A$ free boundary problem and an extension of Muskat model, Acta Math. 122 (1969), 273-300.

20. S. L. Kamenomostskaja, On Stefan's problem, Mat. Sb. 53 (95) (1961), 489514. (Russian) MR $25 \# 5292$.

21. A. Friedman, The Stefan problem in several space variables, Trans. Amer. Math. Soc. 133 (1968), 51-87; Correction, 142 (1969), 557. MR 37 \#3209.

NorthWESTERN University, Evanston, IllinoIs 60201 